baren, virusspezifischen, biologischen Testen nicht erfaßt werden kann. Spätestens 2 Stdn. p.i. ist die Synthese dieses hypothetischen Matrizen-Komplexes abgeschlossen. Nunmehr beginnt die gleichzeitige Synthese von Virus-RNS und s-Antigen-Protein im Zellkern ${ }^{4}$. An diesem Ribonucleoproteid wird sofort das Protein des Hämagglutinins synthetisiert (vgl. auch Anm. ${ }^{28}$ ), das allerdings erst später im Cytoplasma ${ }^{4}$ seine für die biologische Aktivität erforder-

28 L. Hoyle, J. of Hyg. 50, 229 [1952]. liche Struktur erhält. Der Zusammenbau der beiden Untereinheiten zum infektiösen Viruspartikel erfolgt in unmittelbarer Nähe der Zellmembran ${ }^{29}$.

Herrn Professor Dr. W. SснӓғER danken wir für die Unterstützung und Förderung der Arbeit, Fräulein D. Kunert, Herrn O. Harzer und Herrn W. Kraus für ihre wertvolle Mithilfe. Die Arbeit wurde mit Mitteln der Deutschen Forschungsgemeins chaft durchgeführt.

29 G. Нотz u. W. Schäfer, Z. Naturforschg. 10 b, 1 [1955].

\title{
Säurehydrolyse von Ribonucleinsäure und Desoxyribonucleinsäure
}

\author{
Von W. Pollmann und G. Schramm \\ Aus dem Max-Planck-Institut für Virusforschung, Tübingen \\ (Z. Naturforschg. 16 b, 673-678 [1961] ; eingegangen am 28. Juli 1961)
}

\begin{abstract}
A method for the potentiometric titration of secondary phosphate groups in nucleic acids is described. Ribonucleic acids of yeast and of microsomes contain $5-6 \%$ secondary phosphate groups which cannot be removed by dialysis. The potentiometric method was applied to study several enzy. matic hydrolyses and the non-enzymatic hydrolysis between $p_{\mathrm{H}} 2.4-1.8$. The rate of hydrolysis for the purines and for the phosphate groups is approximately proportional to the $\mathrm{H}^{\oplus}$-concentration. The constants of hydrolysis for ribonucleic acid and for deoxyribonucleic acid were determined. In DNA the depurinisation is 650 times faster than in RNA.
\end{abstract}

In den Nucleinsäuren kommen zwei Arten von Bindungen vor, die in saurer Lösung leicht hydrolysiert werden: 1. die glykosidische Bindung zwischen den Zuckerresten und den Purinen und 2. die Phosphorsäurediester-Bindung zwischen den einzelnen Zuckerresten. Die Stabilität der glykosidischen Bindung ist abhängig von den benachbarten Substituenten, die Spaltung erfolgt bei den Purinen bedeutend leichter als bei den Pyrimidinen und ist auch von Purin zu Purin etwas verschieden. Die Gründe hierfür wurden kürzlich in einer Arbeit von Micheel ${ }^{1}$ aufgezeigt. Die Beständigkeit der Glykosid-Bindung ist aber auch von der Art des beteiligten Zuckers abhängig, denn bei der DNS (Desoxyribonucleinsäure) erfolgt die Purinabspaltung viel leichter als bei der RNS (Ribonucleinsäure), so daß aus der DNS Purinreste quantitativ abgespalten werden können, ohne daß hierbei die hochmolekulare Struktur völlig zerstört wird. Diese Hydrolysenprodukte wurden von Chargaff als Apurinsäuren ${ }^{2}$ bezeichnet. Von Freese ${ }^{3}$ wurde gezeigt, daß die Entfernung bestimmter Basen aus der DNS zu Mutationen füh-

1 F. Micheel, A. Hessing, Chem. Ber. 94, 1814 [1961].

2 Ch. Tamm, M. E. Hodes u. E. Chargaff, J. biol. Chemistry 195, 49 [1952]. ren kann, da bei der nachfolgenden Vermehrung diese Fehlstellen durch andere Basen wieder aufgefüllt werden. Auf der anderen Seite geht aus Untersuchungen an verschiedenen isolierten Nucleinsäuren hervor, daß die Hydrolyse der Phosphatbrücken zu einer Inaktivierung führt. Es ist daher wichtig, genaue Unterlagen dafür zu besitzen, inwieweit eine Spaltung der glykosidischen Bindungen zu den Purinen ohne Kettenabbau möglich ist. Die Möglichkeit zur Herstellung von Apurinsäuren hängt von dem Verhältnis der beiden Reaktionskonstanten ab. In der vorliegenden Arbeit wurden daher die Hydrolysen-Geschwindigkeit der Phosphorsäurediester-Gruppen zu den sekundären Phosphaten und die Hydrolysen-Geschwindigkeit der glykosidischen Bindungen $\mathrm{zu}$ den Purinen bei verschiedenen $\mathrm{Nu}$ cleinsäuren quantitativ bestimmt. Zur Messung der ersten Reaktion arbeiteten wir ein potentiometrisches Titrationsverfahren aus. Die Menge der abgespaltenen Purinreste wurde nach bereits bekannten Verfahren durch Chromatographie und anschließende Extinktionsmessung bestimmt.

3 E. Bautz u. E. Freese, Proc. nat. Acad. Sci. USA 47, 540 [1961] ; E. Freese, Brookhaven Sympos. 12, 63 [1959]. 
Die Potentiometrische Titration

Nach F Fetcher ${ }^{4}$ und anderen liegt der $p_{\mathrm{K}}$. Wert der primären Phosphatgruppen in den Nucleinsäuren bei 1, der der sekundären bei 6 . Diese Gruppen sollten also ohne weiteres nebeneinander titrierbar sein. Nach diesen Autoren ist in dem Umschlagsintervall der sekundären Phosphatgruppen keine Störung durch andere dissoziierende Gruppen zu erwarten. Wir überzeugten uns zunächst durch Modellversuche von der Richtigkeit dieser Annahme. Hierzu wurden Mischungen der vier Nucleotide und der vier Nucleoside in den gleichen Mengenverhältnissen hergestellt, wie sie in der Hefe-RNS vorliegen, und von jeder dieser Mischungen die Titrationskurve mit dem Titrator der Firma Radiometer, Copenhagen, aufgenommen. Das Ergebnis ist in Abb. 1 wiedergegeben. Die Mischungen unterscheiden sich nur durch eine Phosphatgruppe je Mol Nucleosid.

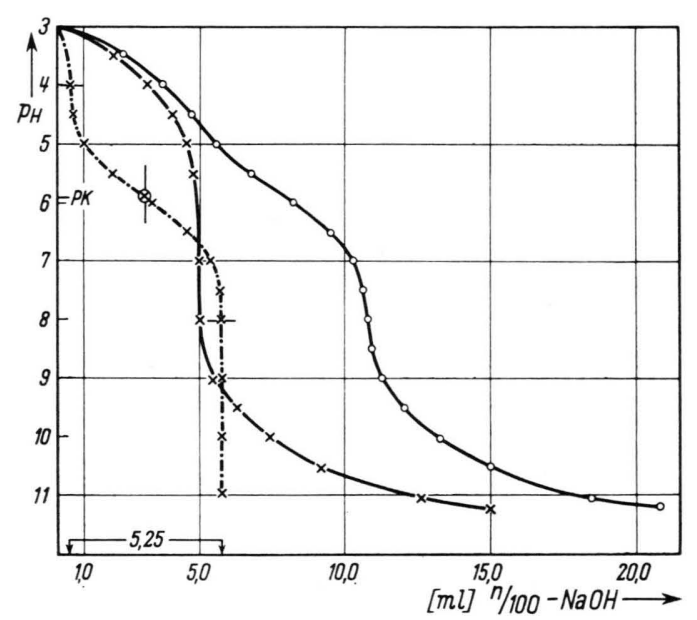

Abb. 1. Titrationskurve des Nucleotid- und Nucleosid-Gemisches bezogen auf $16 \mathrm{mg}$ RNS. Nucleotid-Kurve $0-0$, Nucleosid-Kurve $x \longrightarrow$, Differenz-Kurve $x-\cdot-x$.

Durch die Differenzbildung zwischen Nucleotid- und Nucleosid-Kurve erhält man den exakten Kurvenverlauf für die Dissoziation der Phosphatgruppen. Die Kurve hat bei den $p_{\mathrm{H}^{-}}$Werten 4,0 und 8,05 ihre Wendepunkte. Diese lassen sich besonders genau bestimmen, wenn man die Differenzkurve $\Delta p_{\mathrm{H}}$ : Laugenverbrauch aufträgt. Der Kurvenverlauf zwischen den Wendepunkten entspricht also der Dissoziationskurve der sekundären Phosphatgruppen. Man erkennt weiter, daß zwischen $p_{\mathrm{H}} 3$ und 4 kaum Lauge verbraucht wird, daß also bei dem gewählten Start-

4 W. E. Fletcher, J. M. Gulland u. D. O. Jordan, J. chem. Soc. [London] 1944, 33. punkt die Dissoziation der primären Phosphatgruppe bereits vollständig ist. Der Verlauf der Differenzkurve an den Endpunkten bedingt, daß kleine Meßfehler im $p_{\mathrm{H}}$-Wert das Resultat kaum beeinflussen. Der begrenzende Faktor ist in erster Linie der Bürettenfehler. Er beträgt maximal $0,04 \mathrm{ml} n / 100$ $\mathrm{NaOH}$, was etwa $0,4 \mathrm{mg}$ Mononucleotid entspricht, macht also bei der angewandten Menge von $16 \mathrm{mg}$ maximal $2 \%$ aus. Die Methode ist daher für verhältnismäßig kleine Substanzmengen geeignet.

Anschließend wurde eine vergleichende Titration verschiedener hochmolekularer Ribonucleinsäuren aus Hefe- sowie Ratten- bzw. Kalbslebermikrosomen und eines entsprechenden Gemisches aus Nucleosiden durchgeführt. Falls die hochmolekularen $\mathrm{Nu}$ cleinsäuren nur primäre und keine sekundären Phosphatgruppen enthalten, sollten die Titrationskurven im Bereich $p_{\mathrm{H}} 4-8$ identisch sein. Bei allen drei Nucleinsäure-Präparaten lag aber die Titrationskurve in dem Intervall $p_{\mathrm{H}} 4-8$ um 5-6\% höher (s. Abb. 2). Aus den in der Ultrazentrifuge bestimmten Mol.-Gew. läßt sich berechnen, daß die Zahl der endständigen, sekundären Phosphatgruppen viel geringer als $5 \%$ ist. Alle drei RNS-Präparate haben einen höheren Phosphor-Gehalt als der

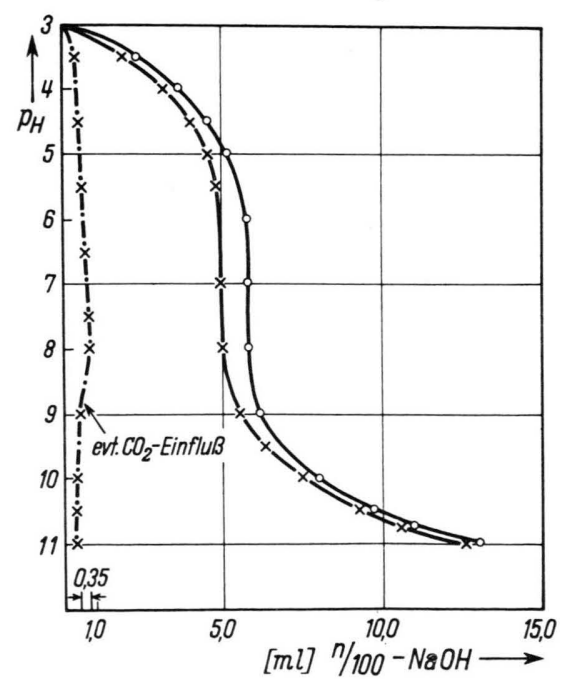

Abb. 2. Titrationskurve von Hefe-RNS und des entsprechenden Nucleosid-Gemisches, bezogen auf $16 \mathrm{mg}$ RNS. HefeRNS-Kurve $0-0$, Nucleosid-Kurve $\times-x$, Differenz-Kurve $x-\cdot-x$.

Theorie entspricht (s. Tab. 2). Dieses Phosphat läßt sich durch Dialyse nicht entfernen. Das Titrationsergebnis ist daher wohl so zu erklären, daß an bestimmten Stellen der Polynucleotidkette zusätzlich Phosphorsäure eingebaut ist. Sekundäre Phosphat- 
gruppen wurden auch von anderen Autoren ${ }^{4-6}$ in der RNS festgestellt. Es scheint uns jedoch nicht gerechtfertigt, hieraus den Schluß zu ziehen, daß diese Polynucleotide eine verzweigte Struktur aufweisen.

Die Titrationskurve von Thymus-DNS wurde ebenfalls mit der eines entsprechenden NucleosidGemisches verglichen. Auch hier ergab sich eine kleine Abweichung, die aber nicht der reinen Titrationskurve einer Phosphatgruppe entsprach. Es dürfte wohl hier die puffernde Wirkung der $\mathrm{H}$ Brücken in der Doppelspirale eine Rolle spielen. Sichere Schlüsse auf das Vorhandensein sekundärer Phosphatgruppen lassen sich hier also nicht ziehen. Mit dem Titrationsverfahren kann jedoch auch bei DNS die Zunahme der sekundären Phosphatgruppen bei hydrolytischen Prozessen gut erfaßt werden.

$$
\text { Enzymatische Hydrolyse }
$$

$\mathrm{Zu}$ Vergleichszwecken wurden zunächst einige enzymatische Hydrolysen ausgeführt. Hierbei wurden die Nucleinsäuren bei konstantem $p_{\mathrm{H}}$-Wert und $37{ }^{\circ} \mathrm{C}$ mit dem entsprechenden Enzym behandelt, zu bestimmten Zeitpunkten Proben entnommen und in diesen der Verbrauch an $n / 100-\mathrm{NaOH}$ zwischen $p_{\mathrm{H}} 4,00$ und 8,00 bestimmt. Am Schluß des Versuchs wurde jeweils eine vollständige Titrationskurve des Spaltgemisches aufgenommen.

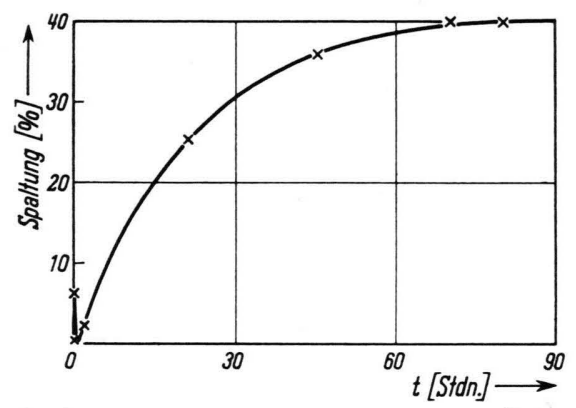

Abb. 3. Spaltung der Hefe-RNS mit Pankreas-Ribonuclease $25,5 \mathrm{mg}$ RNS und $25 \gamma$ Ribonuclease in $100 \mathrm{ml} \mathrm{H}_{2} \mathrm{O}$ bei $p_{\mathrm{H}} 6,5$ und $37^{\circ} \mathrm{C}$.

Der zeitliche Verlauf der Hydrolyse von HefeRNS mit Pankreas-Ribonuclease ist in Abb. 3 wiedergegeben. Da dieses Enzym nur am 3'-Phosphat der Pyrimidin-Nucleotide angreift, ist theoretisch eine 47-proz. Spaltung zu erwarten. Die in Abb. 3 dargestellte Kurve nähert sich asymptotisch einem Spaltendpunkt von 40 Prozent. Vorübergehend sinkt der Wert der sekundären Phosphatgruppen auf Null.

5 L. F. Chavalieri, S. E. Kerr u. A. Angelos, J. Amer. chem. Soc. 73, 2567 [1951].
Dies ist wahrscheinlich durch eine intermediäre Bildung von cyclischem Phosphat zu erklären. Bei dem nach 2 Stdn. Ribonuclease-Einwirkung erhaltenen Oligonucleotid-Gemisch war der $p_{\mathrm{K}}$-Wert geringfügig von 5,9, dem Wert von Mononucleotiden, auf 6,0 verschoben.

Auch bei der Spaltung mit Tabakdiesterase war ein anfängliches Absinken der sekundären Phosphatgruppen zu beobachten. Ein Endwert der Spaltung wurde nach etwa $40 \mathrm{Stdn}$. erreicht, er betrug $87 \%$ des theoretisch zu erwartenden Wertes. Es sind also in der RNS gewisse Bindungen vorhanden, die durch Tabakdiesterase nur langsam angegriffen werden.

Bei der Spaltung von Thymus-DNS mit Desoxyribonuclease wurde nach $10 \mathrm{Stdn}$. ein Endwert erreicht, der $22 \%$ des vorhandenen Phosphats entspricht. Gemäß der allgemeinen Erfahrung wird nur etwa jede vierte Bindung angegriffen.

$$
\text { Säurehydrolyse }
$$

Bei der RNS wurde die Säurehydrolyse der Phosphatgruppen im $p_{\mathrm{H}}$-Bereich $1,8-2,4$ untersucht. In Abb. 4 ist die Zunahme der sekundären Phosphatgruppen in logarithmischem Maßstab aufgetragen.

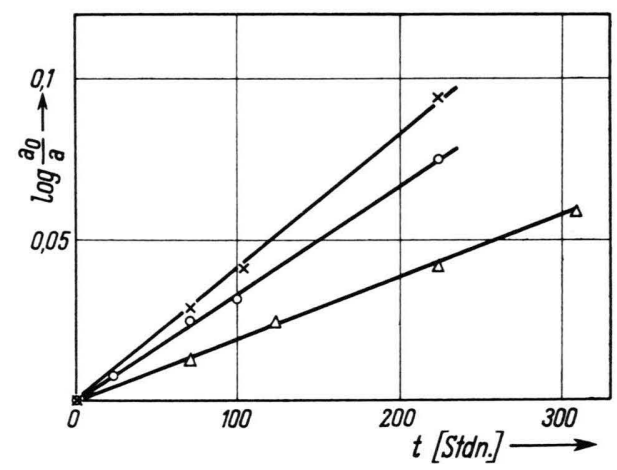

Abb. 4. Säurespaltung der Phosphatbrücken in der Hefe-RNS bei $37^{\circ} \mathrm{C}$. $p_{\mathrm{H}} 1,8_{0} \times ; p_{\mathrm{H}} 2,0_{0} \circ ; p_{\mathrm{H}} 2,4_{0} \triangle$.

Der Kurvenverlauf entspricht einer monomolekularen Reaktion. In der Tab. 1 sind die bei verschiedenen $p_{\mathrm{H}}$-Werten gefundenen Hydrolysekonstanten zusammengestellt. Man erkennt, daß die HydrolysenGeschwindigkeiten der $\mathrm{H}^{\oplus}$-Konzentration annähernd proportional sind. Bemerkenswert ist, daß die Phosphatgruppen bei der DNS etwa gleich schnell hydrolysiert werden wie bei der RNS.

In den gleichen Ansätzen, in denen die Phosphathydrolyse gemessen wurde, wurde auch die Abspal-

6 J. E. Bacher u. F. W. Allan, J. biol. Chemistry 184, 511 [1950]. 


\begin{tabular}{|c|c|c|c|c|c|c|}
\hline \multirow{2}{*}{$p_{\mathrm{H}}$} & \multicolumn{9}{|c|}{ RNS } & \multicolumn{2}{c|}{ DNS } \\
\cline { 2 - 7 } & $\begin{array}{c}K_{\mathrm{Phosphat}} \\
\text { in } 10^{-6} \mathrm{~min}^{-1}\end{array}$ & $\begin{array}{c}K_{\mathrm{Ph}} /(\mathrm{H} \oplus) \\
\text { in } 10^{-3}\end{array}$ & $\begin{array}{c}K_{\text {Purin }} \\
\text { in } 10^{-6} \mathrm{~min}^{-1}\end{array}$ & $\begin{array}{c}K_{\text {Purin }} /(\mathrm{H} \oplus) \\
\text { in } 10^{-4}\end{array}$ & $\begin{array}{c}K_{\text {Phosphat }} \\
\text { in } 10^{-6} \mathrm{~min}^{-1}\end{array}$ & $\begin{array}{c}K_{\mathrm{Purin}} \\
\text { in } 10^{-6} \mathrm{~min}^{-1}\end{array}$ \\
\hline 2,4 & 6,4 & 1,6 & 1,2 & 3,0 & 6,6 & 780 \\
2.0 & 12,7 & 1,3 & 2,7 & 2,7 & - & - \\
1,8 & 15,8 & 1,1 & 3,2 & 3,2 & - & - \\
\hline
\end{tabular}

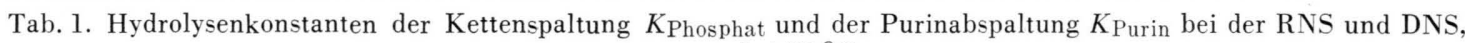
gemessen bei $37^{\circ} \mathrm{C}$.

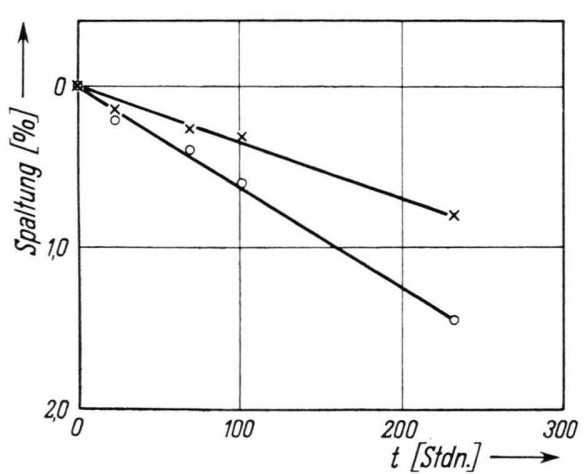

Abb. 5. Basenabspaltung bei Hefe-RNS, $37^{\circ} \mathrm{C}, p_{\mathrm{H}} 1,8_{0}$ Adenin $x ;$ Guanin $O$.

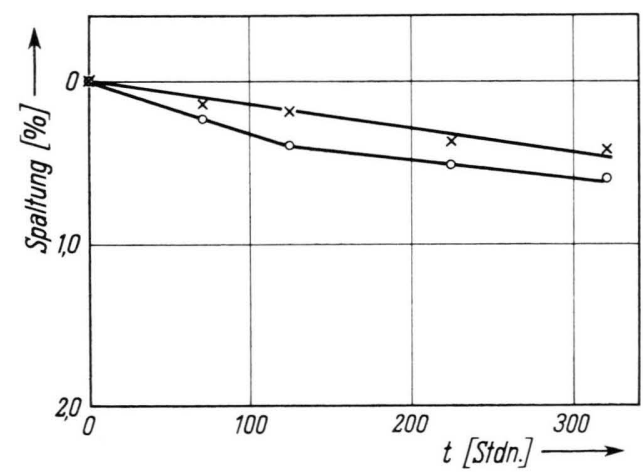

Abb. 6. Basenabspaltung bei Hefe-RNS, $37^{\circ} \mathrm{C}, p_{\mathrm{H}} 2,4_{0}$ Adenin $x$; Guanin $O$.

tung der Basen verfolgt. Zwei Beispiele sind in Abb. 5 und 6 wiedergegeben. Die Abspaltung des Guanins erfolgt bei $p_{\mathrm{H}} 1,8$ etwa doppelt so schnell wie die des Adenins. Bei $p_{\mathrm{H}} 2,4$ ist dies anfangs ebenso, doch sinkt später die Hydrolyse-Geschwindigkeit des Guanins auf den Wert des Adenins. In Tab. 1 sind die mittleren Hydrolysen-Geschwindigkeiten der Purinreste bei verschiedem $p_{\mathrm{H}}$-Wert angegeben; auch die Spaltung der Glykosidbindung erfolgt also etwa proportional der $\mathrm{H}^{\oplus}$-Konzentration. Bei der DNS wurden Adenin und Guanin mit derselben Geschwindigkeit abgespalten, doch sind hier die Hydrolysen-Geschiwndigkeiten der Purinreste 650-mal höher als bei der RNS (s. Tab. 1).

\section{Besprechung der Ergebnisse}

Da sowohl die Phosphatspaltung als auch die Glykosid-Spaltung annähernd der $\mathrm{H}^{\oplus}$-Konzentration proportional ist, ist die Ausbeute an Apurinsäuren unabhängig vom $p_{\mathrm{H}}$-Wert. Die Abhängigkeit der Reaktionsgeschwindigkeiten von der Protonenkonzentration spricht dafür, daß der geschwindigkeitsbestimmende Schritt die Anlagerung eines Protons ist. Sie wird um so langsamer erfolgen, je elektronenärmer der Angriffsort ist. Es ist daher verständlich, daß die elektronen-anziehende $\mathrm{OH}$-Gruppe im $\mathrm{C}^{\prime}{ }^{\prime}$ einen stabilisierenden Einfluß auf die Glykosidbindung ausübt. Bei der DNS gelingt also die Darstellung von Apurinsäure sehr viel leichter als bei RNS. Trotzdem besteht auch hier eine Möglichkeit, intakte Moleküle zu erhalten, die einen Purinrest verloren haben. Der Anteil der Ketten, die nach der Zeit $t$ noch keine Phosphatspaltung, jedoch eine Purinspaltung, erlitten haben, ist

$$
M_{\text {Apurinsäure }}=e^{-N_{1} \alpha t}\left(1-e^{-N_{2} \beta t}\right)
$$

$N_{1}=$ Zahl der Phosphatbrücken $=$ Zahl der Kettenglieder; $\alpha=k_{\text {Phosphat }} ; \quad \beta=k_{\text {Purin }} ; \quad N_{2}=$ Zahl der Purinreste.

Diese Funktion hat ein Maximum bei

$$
t=\ln \frac{N_{1} \alpha+N_{2} \beta}{N_{1} \alpha}: N_{2} \beta .
$$

Für die RNS aus Tabakmosaikvirus gelten folgende Werte $N_{1}=6400, N_{2}=3200$. Bei einem $p_{\mathrm{H}}$ von 2,4 und $37{ }^{\circ} \mathrm{C}$ ist dann $t_{\max }=25$ Minuten. Durch Einsetzen dieser Zahl in Gl. (1) erhält man den maximalen Anteil an Apurinsäuren zu $M_{\mathrm{A}}=3,6$ Prozent. $\mathrm{Zu}$ diesem Zeitpunkt ist die Zahl der noch vollkommen intakten Moleküle, die weder Phosphat- noch Purinspaltung erlitten haben, 33\% und die Zahl der bereits gespaltenen Moleküle 63 Prozent. 
Bei der DNS verkürzt sich $t_{\max }$ infolge der schnelleren Purinspaltung und des höheren Mol.-Gew. natürlich erheblich. Es ist hier verhältnismäßig leicht, Ketten zu erhalten, die mehrere Purinreste verloren haben, ohne daß sie zerbrochen sind. Auf die Wiedergabe einer Berechnung wird hier verzichtet. Es wird interessant sein zu prüfen, wieweit derartige Apurinsäuren in ihrer genetischen Funktion verändert sind. Von Schramm, Grötsch und PollMANN $^{7}$ wurde weiterhin ein Verfahren gefunden, um die entstandenen Fehlstellen durch andersartige Basen wieder aufzufüllen. Es können also nach der Nomenklatur von Fre es e verschiedene Transitionen (Ersatz eines Purins durch ein anderes Purin) und Transversionen (Ersatz eines Purins durch ein Pyrimidin) durchgeführt werden.

\section{Material und Methoden}

\section{Darstellung der Nucleinsäuren}

Die RNS aus Bäckerhefe wurde in Anlehnung an die Vorschrift von Chesterfield ${ }^{8}$ mit Hilfe von Natriumdodecylsulfat und mehrfacher NaCl-Fällung hergestellt. Die Mikrosomen-RNS aus Ratten- bzw. Kalbsleber wurde aus der durch differentielle Zentrifugation der Leberhomogenate ${ }^{9}$ gewonnenen Mikrosomenfraktion isoliert. Hierzu wurde die Phenol-Extraktionsmethode von Schuster, Schramm und $Z_{\text {Illig }}{ }^{10}$ und anschließende Äthanolfällung angewandt. Die Darstellung der DNS erfolgte nach $Z_{\text {AMENHOF }}{ }^{11}$ aus frischen Kalbsthymusdrüsen mit Natriumcitratpuffer sowie NaCl- und Äthanolfällungen.

\begin{tabular}{|l|c|c|c|}
\hline & Hefe-RNS & $\begin{array}{c}\text { Mikroso- } \\
\text { men-RNS }\end{array}$ & $\begin{array}{c}\text { Thymus- } \\
\text { DNS }\end{array}$ \\
\hline DNS-Gehalt & $0,5 \%$ & $0,27 \%$ & - \\
Protein-Gehalt & $1 \%$ & $1 \%$ & $1 \%$ \\
Phosphor & $8,2 \%$ & $9,7 \%$ & $9,8 \%$ \\
$\begin{array}{l}\text { Stickstoff } \\
\text { Atom. N/P }\end{array}$ & $13,4 \%$ & $16,6 \%$ & $18,6 \%$ \\
$\begin{array}{l}\text { Viskosität }[\eta] \\
0,02-m \cdot \text { Phosphat }\end{array}$ & 3,6 & 3,8 & 4,1 \\
$p_{\text {H 7 }}$ & & & \\
$\begin{array}{l}\text { Sedimentations- } \\
\text { konstante in S }\end{array}$ & 65 & 103 & ca. $10^{3}$ \\
Molekulargewicht & 65000 & $28 ; 17 \mathrm{~S}$ & - \\
\hline & & $0,6 \cdot 10^{6}$ & $6 \cdot 10^{6}$ \\
\hline
\end{tabular}

Tab. 2. Charakterisierung der Nucleinsäure-Präparate.

7 G. Schramm, H. Grötsch u. W. Pollmann, Angew. Chem., im Druck.

8 A. M. Chestfield, K. C. Smith u. F. W. Allan, J. biol. Chemistry 216, 185 [1955].

9 J. W. Littlefield, E. B. Keller, J. Gross u. P. C. Zamecnik, J. biol. Chemistry 271, 111 [1955].
Reinheitsprüfung der Nucleinsäuren

Zur Bestimmung des DNS-Gehalts in den RNS-Präparationen benützten wir die Methode nach Dische ${ }^{13}$ mittels Diphenylamin. Der Proteingehalt wurde nach der verfeinerten Biuret-Reaktion nach Markham ${ }^{14}$ bestimmt. Die Viskositätsmessungen erfolgten im $\mathrm{Ost}$. w a ld - Kapillar-Viskosimeter bei $20,0^{\circ} \mathrm{C}$. Durch Messungen an Verdünnungsreihen konnte auf die Viskositätszahl $[\eta]_{c \rightarrow 0}$ extrapoliert werden. Die Eigenschaften der verschiedenen Präparate sind in Tab. 2 zusammengestellt.

\section{Potentiometrische Titration}

Die vorliegenden Titrationen wurden mit dem Autotitrator der Firma Radiometer, $p_{\mathrm{H}}$-Genauigkeit 0,02 Einheiten, durchgeführt. Titriert wurde bei $20,0{ }^{\circ} \mathrm{C}$ unter magnetischem Rühren und Stickstoff mit $n / 100$ $\mathrm{NaOH}$ aus einer Feinbürette mit einer Ablesegenauigkeit von 0,02 Milliliter. Die Eichung erfolgte jeweils mit Standard-Acetat-Puffer von $p_{\mathrm{H}} 4,62$. Für die vollständigen Titrationskurven wurde der Verbrauch an $n / 100-\mathrm{NaOH}$ in $p_{\mathrm{H}}$-Intervallen von 0,5 Einheiten gemessen. Bei den Spaltungsversuchen wurde die Probe auf $p_{\mathrm{H}} 4$ gebracht und der Gesamtverbrauch zwischen $p_{\mathrm{H}} 4,0$ und 8,0 bestimmt.

$$
\text { Messung der Purin-Abspaltung }
$$

$\mathrm{Zu}$ bestimmten Zeitabständen entnommene Proben wurden gefriergetrocknet, in $2 \mathrm{ml}$ Wasser aufgenommen und in zwei Parallelbestimmungen das freigesetzte Adenin und Guanin auf Whatman Nr. 1-Papier chromatographisch abgetrennt. Wir benützten hierbei eine Mischung von n-Butanol, Äthylenglykol und Wasser im Verhältnis $4: 1: 1$. Die hochmolekularen Nucleinsäuren und die Oligonucleotide wandern in diesem System nicht. Der $R_{f}$-Wert für Adenin betrug 0,52, für Guanin 0,23 und schwankte nicht mehr als um 0,02 Einheiten. Die im Ultraviolett-Licht gut sichtbaren Flecken wurden ausgeschnitten und mit $n / 10-\mathrm{HCl} 45 \mathrm{Min}$. bei $45^{\circ} \mathrm{C}$ eluiert. Die Konzentration wurde bei $p_{\mathrm{H}} 2,0$ aus der Extinktion bei $260 \mathrm{~m} \mu$ bestimmt. Hierbei wurden folgende Extinktionswerte zugrunde gelegt: Adenin je $1 \mathrm{mMol} / l 13,0$; Guanin 8,0.

$$
\text { Hydrolyse der Nucleinsäuren }
$$

$\mathrm{Zu}$ den Ansätzen wurden jeweils einige Tropfen Chloroform hinzugegeben, um eventuelles Wachstum von Mikroorganismen und damit erhöhten Nucleinsäureabbau zu verhindern. Die Teste auf Mikroorganismen in den Ansätzen mit Agar-Agar-Platten verliefen stets negativ. Auch die Kontrollversuche auf Nucleasen bei $p_{\mathrm{H}} 7$ erbrachten keinen nennenswerten Abbau der Nucleinsäuren.

10 H. Schuster, G. Schramm u. W. Zillig, Z. Naturforschg. 11 b, 339 [1956].

11 W. Frisch-Niggemeyer u. K. K. Reddi, Biochim. biophysica Acta [Amsterdam] 26, 40 [1957].

12 S. Zamenhof, Methods in Enzymology III, S. 698.

13 Z. Dische, Z. Mikrochem. 8, 4 [1930].

14 R. Markham u. J. D. Sмith, Biochem. J. 46, 509 [1950]. 


$$
\text { Enzyme }
$$

Als Pankreas-Ribonuclease diente ein 5-fach umkristallisiertes Präparat der Firma Pentex, Illinois. Die Tabakdiesterase wurde nach der Methode von
Frisch-Niggemeyer und Reddi ${ }^{11}$ von Herrn Dipl.-Chemiker Süss hergestellt, wofür wir ihm vielmals danken. $\mathrm{Als}$ Desoxyribonuclease verwandten wir ein Präparat von Worthington Biochemical Corporation, Freehold, New Jersey.

\title{
Leudhtbakterien als Ausgangsprodukt für Isocitratase
}

\author{
Von A. F. Kremmeter, H. J. Schliep und F. Bukatsch \\ Aus dem Botanischen Institut (Direktor Prof. Dr. phil. L. BRAuner) \\ und dem Strahlenbiologischen Institut der Universität München (Direktor Prof. Dr. med. O. Hug)
}

(Z. Naturforschg. 16 b, 678-680 [1961]; eingegangen am 14. Juli 1961)

\begin{abstract}
Die beschriebene Isolierung von Leuchtbakterien-Isocitratase und die methodisch einfache Züchtung von Photobacterium fischeri in submerser Kultur erlauben die Darstellung von Isocitratase im großen Ausmaß. Mit Hilfe der isoelektrischen Fällung eines wäßrigen Extraktes aus Acetontrockenpulver und anschließender Ammonsulfat-Fraktionierung läßt sich bei 2,05-m. Ammonsulfat-Konzentration Isocitratase aus Leuchtbakterien gewinnen. Das unter $\mathrm{N}_{2}$-Atmosphäre einige Tage stabile Ferment konnte 30 -fach angereichert werden.
\end{abstract}

Isocitratase, ein von Campbell ${ }^{1}$ in Pseudomonas aeruginosa gefundenes Enzym, wurde im Rahmen des „By-pass“ von Kornberg ${ }^{2}$ und Mitarbb. näher untersucht. 1960 konnten Kremmeter und Bukatsch ${ }^{3,4}$ den Glyoxylsäurezyklus auch in Photobacterium fischeri nachweisen; im Gegensatz zu Pseudomonas findet sich die Leuchtbakterien-Isocitratase auch in Kulturen, die in acetatfreiem Medium gezogen wurden; das Enzym ist also nicht ausschließlich Substrat induziert. Es benötigt neben $\mathrm{Mg}^{2 \oplus}$-Ionen Cystein als Aktivator. In der vorliegenden Arbeit wird ein relativ unkompliziertes Anreicherungsverfahren der Isocitratase beschrieben, die sich aus Leuchtbakterien leicht in großen Mengen darstellen läßt.

\section{Methodik}

Photobact. fischeri wurde in 20-l-Ansätzen * gezüchtet und aus der $60 \mathrm{Stdn}$. nach Inkubation durch Abzentrifugieren gewonnenen Bakterienmasse ein Acetontrockenpulver ${ }^{5}$ hergestellt.

Nach mechanischer Aufbereitung der 25-proz. wäß. rigen Suspension des Pulvers im Zellhomogenisator von

1 J. J. R. Campbell, R. A. Smith a. B. A. Eagles, Biochim. biophysica Acta [Amsterdam] 11, 594 [1953].

2 H. L. Kornberg, Fourth International Congress of Biochemistry, 13, 251 [1958].

3 A. F. Kremmeter u. F. Bukatsch, Naturwissenschaften 47, 500 [1960].

1 A. F. Kremmeter u. F. Bukatsch, im Druck.

* Das verwendete Kulturmedium hatte folgende Zusammen. setzung: $30,0 \mathrm{~g} \mathrm{NaCl}, 2,6 \mathrm{~g} \mathrm{Na}_{2} \mathrm{HPO}_{4} \cdot 2 \mathrm{H}_{2} \mathrm{O}, 2,1 \mathrm{~g} \mathrm{KH}_{2} \mathrm{PO}_{4}$, $0,5 \mathrm{~g}_{\left(\mathrm{NH}_{4}\right)} \mathrm{HPO}_{4}, 0,1 \mathrm{~g} \mathrm{MgSO}_{4}, 1,0 \mathrm{~g}$ Pepton, $6 \mathrm{ml}$ Glycerin und dest. Wasser ad $1000 \mathrm{ml}$.
Merkenschlager ${ }^{6}$ wurde der Eiweißextrakt bei ca. $25000 \mathrm{~g}$ in der Kälte klarzentrifugiert und durch $\mathrm{Zu}$ satz von $n / 10-\mathrm{HCl}$ im Bereich von $p_{\mathrm{H}} 4,5-4,8$ gefällt ${ }^{3}$. Zugabe von festem $\left(\mathrm{NH}_{4}\right)_{2} \mathrm{SO}_{4}$ in die Lösung des aufgenommenen isoelektrischen Präzipitates lieferte Unterfraktionen bei 33-, 50-, 65-, 71- und 100-proz. Sättigung der Lösung. Diese Bereiche hatten sich bereits während der Anreicherung der Bakterien-Luciferase für die Röntgenbestrahlung isolierter Fermente als günstig erwiesen $\left(\right.$ Schliep $^{7}$ ), die eine Fortsetzung der reaktionskinetischen Untersuchungen ${ }^{8}$ an intakten Bakterien darstellen. Die einzelnen Ammonsulfatfällungen wurden niedertourig abzentrifugiert und unter gutem Rühren in $0,02-m$. Kalium-Phosphatpuffer $p_{\mathrm{H}} 7,0$ aufgenommen. In allen Arbeitsgängen diente $\mathrm{N}_{2}$ als Schutzgas. Die Bestimmung der Eiweißkonzentrationen erfolgte mit der von Frank und Koecher ${ }^{9}$ modifizierten Weichselbaum-Biuret-Methode.

Die reversible Aldolspaltung des Isocitrats zu Succinat und Glyoxylat in Gegenwart des Enzyms wurde mit dem Spektralphotometer PMQ II (Zeiss) nach dem Verfahren von Dixon und Kornberg ${ }^{10}$ bestimmt (Messung der zunehmenden optischen Dichte bei $324 \mathrm{~m} \mu$ in $\mathrm{Ab}$ hängigkeit von der Menge des entstehenden Glyoxylsäurephenylhydrazons). In Abänderung der angegebenen Methode lief die Reaktion bei $p_{\mathrm{H}} 7,4 \mathrm{ab}$, da in diesem Bereich ein erhöhter Umsatz erzielt wird ${ }^{4}$; außerdem setzten wir statt 6,0 nur 2,0 $\mu$ Mole Cystein ein, nach-

5 B. L. Strehler a. M. J. Cormier, Arch. Biochem. Biophysics 47, 16 [1953].

6 M. Merkenschlager, K. Schlossmann u. W. Kurz, Biochem. Z. 329, 332 [1957].

7 H. J. Schliep, in Vorbereitung.

8 D. Harder u. O. Hug, Strahlentherapie 106, 245 [1958].

9 H. Frank u. P. H. Koenler, Dtsch. Arch. klin. Med. 197. 181 [1950].

10 G. H. Dixon a. H. L. Konnberg, Biochem. J. 72, 3 [1959]. 